\title{
Modeling diameter distribution of the broadleaved-Korean pine mixed forest on Changbai Mountains of China
}

\author{
WANG Shunzhong ${ }^{1,2}$, DAI Limin ${ }^{1}$, LIU Guohua ${ }^{3}$, YUAN Jianqiong ${ }^{1,2}$, \\ ZHANG Hengmin ${ }^{1,2} \&$ WANG Qingli ${ }^{1}$ \\ 1. Institute of Applied Ecology, Chinese Academy of Sciences, Shenyang 110016, China; \\ 2. Graduate University of Chinese Academy of Sciences, Beijing 100039, China; \\ 3. State Key Lab of Systems Ecology, Research Center for Eco-Environmental Sciences, Chinese Acad- \\ emy of Sciences, Beijing 100085, China \\ Correspondence should be addressed to Liu Guohua (email: ghliu@rcees.ac.cn) \\ Received October 10, 2005; accepted April 12, 2006
}

\begin{abstract}
The broadleaved-Korean pine mixed forest is a native vegetation in the Changbai Mountains, northeast China. The probability density functions including the normal, negative exponential, Weibull and finite mixture distribution, were used to describe the diameter distributions of the species groups and entire forest stand. There is a strong correlation between parameters and mean DBH except the shape parameters in the mixture distribution. The diameter classes of species and entire forest stand showed not negative exponential but normal and "S" distribution. The mixture function was better than normal and Weibull to describe the model distribution. The location parameter had an effect on the estimated frequency in the first diameter class, when the estimated location parameter was bigger than the lower limit of the first diameter class.
\end{abstract}

Keywords: broadleaved-Korean pine mixed forest, diameter distribution, finite mixture distribution.

\section{Introduction}

The diameter distribution is a potent and simple factor for depicting the properties of a stand of trees ${ }^{[1]}$ and the central element growth modeling ${ }^{[2]}$. Though the ecological research has focused on better understanding the structure, function, and productivity of broadleaved-Korean pine mixed forest, the diameter distribution has rarely been described $^{[3-6]}$. Modeling diameter distribution of a forest is an important step to develop optimal forest management schemes. Therefore, it is necessary to model diameter distributions of broadleaved-Korean pine mixed forest stands.

For an uneven-aged mixed forest stand, the diameter distribution consists of stand- 
level and special-level diameter distributions. Meyer and Stevenson ${ }^{[7]}$ applied the exponential distribution to mixed forests in Pennsylvania, United States. Meyer ${ }^{[8]}$, Schmelz and Lindsey ${ }^{[9]}$ found the exponential function satisfactory to other forest stands. Leak ${ }^{[10]}$ modified this kind of modeling by using reversed J-shaped functions. The Weibull function, developed by Weibull ${ }^{[11]}$ and introduced by Bailey and Dell ${ }^{[1]}$, is one of the most popular functions because of its relative simplicity on estimations of parameter and its flexibility for various shapes and skewness extents. Because the Weibull function is a unimodal curve, the finite mixture distribution is developed in the "rotated-sigmoid" forest stand defined by Goff and West ${ }^{[12]}$. Zhang ${ }^{[13]}$ and Liu ${ }^{[14]}$ were pioneers who successfully used the finite mixture model in mixed-species forest stands. Special-level diameter distributions have been developed in Scots pine and mixed spruce-fir forest stand, however, they were rarely used to deal with an uneven-aged mixed multi-species forest stand $^{[14,15]}$.

The objective of this study was to investigate the suitabilities of various functions such as the exponential, the Weibull function, and the finite mixture function in modeling the diameter distributions of a typical broadleaved-Korean pine mixed forest stand on the Changbai Mountains in Northeastern China.

\section{Material and methods}

\subsection{Study area}

The Changbai Mountains are located along the China-North Korea border. In the Changbai Mountains area, typical broadleaved-Korean pine mixed forest is found out at the altitude between $700-1100 \mathrm{~m}$, which covered an area of 42,000 ha within the Changbai Mountains Nature Reserve. The climate in this vegetation zone is characterized by long and cold winter and short and cool summers. The monthly mean temperature ranges from $-18^{\circ} \mathrm{C}$ in January to $18^{\circ} \mathrm{C}$ in July. The accumulated temperature above $10^{\circ} \mathrm{C}$ is about $1500^{\circ} \mathrm{C}$. The annual precipitation is $700-800 \mathrm{~mm}$ and frost-free duration is $100-200$ days. The soil type is a dark-brown forest soil.

\subsection{Sampling}

The study plot is located in the north slope of Changbai Mountains. It was originally set up in 1981 by the Changbai Mountains Forest Ecosystem Research Station, Chinese Academy of Sciences. At an elevation of $740 \mathrm{~m}$, the size of the plot was $100 \mathrm{~m} \times 100 \mathrm{~m}$. In 1992, the diameter at breast height of trees $(\mathrm{DBH}>8 \mathrm{~cm})$ within this plot was measured with the diameter tape to the nearest millimeter. There were a total of 492 trees of greater than $8 \mathrm{~cm}$ in DBH in the plot.

\subsection{Data analysis}

There are fifteen species in the plot. In the species-level distributions, twelve species were categorized into seven groups in terms of species/genus: Tilia sp. (S1), Pinus koraiensis (S2), Acer sp. (S3), Fraxinus mandshurica (S4), Ulmus sp.(S5), Sophora sp. (S6), Quercus mongolica (S7), while three species including Phellidendron amurense, 
Syringa amurensis and Betula platyphylla were removed since the number of trees was too small. These fifteen species also were grouped into canopy tree species (CTS) including P. Koraiensi, T. amurensis, and F. mandshurica vs. sub-canopy tree species (SCTS), and pine (S2) vs. broadleaved tree species (BTS), based on the species' characteristics.

This study intended to model the diameter distribution of the stand with five functions, including normal distribution (eq. (1)), negative exponential function (eq. (2)), Weibull function (eq. (3)), and two finite mixture models (eqs. (4), (5)).

$$
f(x, \theta)=\frac{1}{\sqrt{2 \pi} \sigma} \exp \left(-\frac{1}{2 \sigma^{2}}(x-\mu)^{2}\right),
$$

where $\theta=(\sigma, \mu)^{\prime}$ and $\mu$ and $\sigma$ are the mean and standard deviation.

$$
f(x, \theta)=\left(\frac{1}{\beta}\right) \mathrm{e}^{-(x-\alpha) / \beta},
$$

where $\theta=(\alpha, \beta)^{\prime}$ and $\alpha$ and $\beta$ are the location and scale.

$$
f(x, \theta)=\left(\frac{\gamma}{\beta}\right)\left(\frac{x-\alpha}{\beta}\right)^{\gamma-1} \mathrm{e}^{-[(x-\alpha) / \beta]^{\gamma}} \quad(\infty>x \geqslant \alpha, \beta>0, \gamma>0),
$$

where $\theta=(\alpha, \beta, \gamma)^{\prime}$ and $\alpha, \beta$, and $\gamma$ are the location, scale, and shape parameters, respectively.

$$
\begin{array}{r}
f(x, \psi)=p_{1} f_{1}\left(x, \theta_{1}\right)+\left(1-p_{1}\right) f_{2}\left(x, \theta_{2}\right), \\
0 \leqslant p_{1} \leqslant 1, f_{i}\left(x, \theta_{i}\right)=\left(\frac{\gamma_{i}}{\beta_{i}}\right)\left(\frac{x-\alpha_{i}}{\beta_{i}}\right)^{\gamma_{i}-1} \mathrm{e}^{-\left[\left(x-\alpha_{i}\right) / \beta_{i}\right]^{\gamma_{i}}},
\end{array}
$$

where $\psi=\left(\theta_{1}, \theta_{2}\right)$ with $\theta_{i}=\left(\alpha_{i}, \beta_{i}, \gamma_{i}\right)^{\prime}$, and $i=1$ and 2 .

$$
\begin{aligned}
& f(x, \psi)=p_{1} f_{1}\left(x, \theta_{1}\right)+p_{2} f_{2}\left(x, \theta_{2}\right)+\left(1-p_{1}-p_{2}\right) f_{3}\left(x, \theta_{3}\right), \\
& 0 \leqslant p_{i} \leqslant 1, f_{i}\left(x, \theta_{i}\right)=\left(\frac{\gamma_{i}}{\beta_{i}}\right)\left(\frac{x-\alpha_{i}}{\beta_{i}}\right)^{\gamma_{i}-1} \mathrm{e}^{-\left[\left(x-\alpha_{i}\right) / \beta_{i}\right]_{i}^{\gamma_{i}},},
\end{aligned}
$$

where $\psi=\left(\theta_{1}, \theta_{2}, \theta_{2}\right)$ with $\theta_{i}=\left(\alpha_{i}, \beta_{i}, \gamma_{i}\right)^{\prime}$, and $i=1,2$ and 3 .

Eqs. (1) - (4) were utilized to characterize the diameter distributions of the single species/genus, canopy tree species vs. sub-canopy tree species, and pine vs. broadleaved tree species. The diameter distribution of the entire stand of the broadleaved-Korean pine mixed forest was described by eqs. (2)-(5). In these probability density functions, the parameters can be estimated by different statistical methods such as maximum likelihood, moments, and percentiles. The parameters of the probability density functions in this paper were estimated by the maximum likelihood method in soft package Weibull++6.

We used the bias (Bias) and the root mean square error (RMSE) ${ }^{[14]}$ to compare the equations. To evaluate the probability density functions, the likelihood-ratio $\chi^{2}$ was used for testing "goodness of fit" and is computed as 


$$
x^{2}=\sum_{i}^{n} \frac{\left(N_{i}-\hat{N}_{i}\right)^{2}}{\hat{N}_{i}}
$$

where $N_{i}$ is the observed frequency in the $i$ th diameter class, $\hat{N}_{i}$ is the estimated frequency in the $i$ th diameter class, $q$ is the number of estimated parameters. The $\chi^{2}$ has $(n-q-1)$ degrees of freedom.

\section{Results}

\subsection{Estimated parameters}

The parameters, estimated by maximum likelihood with the measured data for each group and entire stand, are shown in Table 1. All parameters were successfully computed except the parameters of S7 in eq. (4). Parameters of the normal function were the

Table 1 Parameter estimates of each group and entire stand with the location parameter considered as the low value in the smallest diameter class (a) and estimated in eqs. (2)-(5) and considered as the same in eqs. (3) - (5) and as zero when the estimated location parameters were negative (b)

(a)

\begin{tabular}{|c|c|c|c|c|c|c|c|c|c|c|c|c|c|}
\hline & \multicolumn{2}{|c|}{ Eq. (1) } & \multicolumn{2}{|c|}{ Eq. (2) } & \multicolumn{3}{|c|}{ Eq.(3) } & \multicolumn{6}{|c|}{ Eq.(4) } \\
\hline & $\mu$ & $\theta$ & $\alpha$ & $\beta$ & $\alpha$ & $\beta$ & $\gamma$ & $\rho$ & $\alpha_{\mathrm{i}}$ & $\beta_{1}$ & $\gamma_{1}$ & $\beta_{2}$ & $\gamma_{2}$ \\
\hline S1 & 32.09 & 16.75 & 8 & 24.10 & 8 & 25.92 & 1.30 & 0.26 & 8 & 4.39 & 1.95 & 35.29 & 2.54 \\
\hline S2 & 31.32 & 10.46 & 12 & 19.31 & 12 & 21.47 & 1.79 & 0.15 & 12 & 6.57 & 0.99 & 24.37 & 2.50 \\
\hline S3 & 18.17 & 7.63 & 8 & 10.17 & 8 & 11.18 & 1.38 & 0.81 & 8 & 8.02 & 1.72 & 24.75 & 5.82 \\
\hline S4 & 51.33 & 13.54 & 16 & 35.34 & 16 & 39.25 & 2.83 & 0.18 & 16 & 18.42 & 1.40 & 43.09 & 4.60 \\
\hline S5 & 27.10 & 14.24 & 8 & 19.08 & 8 & 20.91 & 1.38 & 0.40 & 8 & 6.90 & 2.60 & 31.20 & 2.48 \\
\hline S6 & 12.91 & 3.03 & 8 & 4.91 & 8 & 5.52 & 1.71 & 0.40 & 8 & 2.32 & 2.88 & 7.62 & 3.31 \\
\hline S7 & 49.92 & 12.72 & 16 & 33.90 & 16 & 36.93 & 2.53 & & & & & & \\
\hline CTS & 35.01 & 15.36 & 8 & 20.96 & 8 & 30.60 & 1.55 & 0.08 & 8 & 4.04 & 2.21 & 33.07 & 2.06 \\
\hline SCTS & 21.08 & 12.63 & 8 & 13.07 & 8 & 13.67 & 1.12 & 0.58 & 8 & 6.59 & 1.81 & 25.93 & 1.73 \\
\hline BTS & 27.57 & 17.12 & 8 & 19.57 & 8 & 20.29 & 1.10 & 0.48 & 8 & 6.62 & 1.73 & 36.23 & 2.31 \\
\hline \multirow[t]{3}{*}{ ES } & & & 8 & 20.53 & 8 & 22.06 & 1.26 & 0.33 & 8 & 6.23 & 1.82 & 31.38 & 2.06 \\
\hline & \multicolumn{9}{|c|}{ Eq. (5) } & & & & \\
\hline & $\rho_{1}$ & $\rho_{2}$ & $\alpha_{\mathrm{i}}$ & $\beta_{1}$ & $\gamma_{1}$ & $\beta_{2}$ & $\beta_{2}$ & $\beta_{3}$ & $\gamma_{3}$ & & & & \\
\hline ES & 0.12 & 0.20 & 8 & 2.90 & 2.70 & 7.93 & 3.24 & 31.31 & 2.05 & & & & \\
\hline \multicolumn{14}{|l|}{ (b) } \\
\hline & \multicolumn{2}{|c|}{ Eq. (1) } & \multicolumn{2}{|r|}{ Eq. (2) } & \multicolumn{3}{|c|}{ Eq. (3) } & \multicolumn{6}{|c|}{ Eq. (4) } \\
\hline & $\mu$ & $\theta$ & $\alpha$ & $\beta$ & $\alpha^{*}$ & $\beta$ & $\gamma$ & $\rho$ & $\alpha_{\mathrm{i}}$ & $\beta_{1}$ & $\gamma_{1}$ & $\beta_{2}$ & $\gamma_{2}$ \\
\hline S1 & 32.09 & 16.75 & 9 & 23.09 & 0 & 36.33 & 2.05 & 0.18 & 0 & 11.32 & 11.91 & 41.50 & 2.76 \\
\hline S2 & 31.32 & 10.46 & 12 & 19.23 & 5.85 & 28.70 & 2.66 & 0.88 & 5.85 & 26.15 & 2.84 & 43.43 & 9.63 \\
\hline S3 & 18.17 & 7.63 & 8.5 & 9.67 & 8.42 & 10.57 & 1.29 & 0.83 & 8.42 & 7.83 & 1.52 & 24.87 & 6.35 \\
\hline S4 & 51.33 & 13.54 & 17 & 33.90 & 0 & 56.28 & 4.63 & 0.42 & 0 & 45.41 & 3.83 & 62.43 & 8.41 \\
\hline S5 & 27.10 & 14.24 & 9.8 & 17.30 & 8.95 & 19.47 & 1.25 & 0.40 & 8.95 & 5.78 & 2.06 & 30.22 & 2.42 \\
\hline S6 & 12.91 & 3.03 & 8.9 & 4.01 & 8.71 & 4.55 & 1.33 & 0.42 & 8.71 & 1.59 & 1.60 & 6.94 & 3.03 \\
\hline S7 & 49.92 & 12.72 & 17 & 33.44 & 0 & 54.45 & 4.93 & & & & & & \\
\hline CTS & 35.01 & 15.36 & 9 & 26.04 & 2.44 & 36.83 & 2.27 & 0.09 & 2.44 & 10.95 & 4.98 & 39.22 & 2.65 \\
\hline SCTS & 21.08 & 12.63 & 8.5 & 12.58 & 8.49 & 12.82 & 1.04 & 0.60 & 8.49 & 6.16 & 1.50 & 26.21 & 1.75 \\
\hline BTS & 27.57 & 17.12 & 8.5 & 19.08 & 8.49 & 19.35 & 1.04 & 0.51 & 8.49 & 6.47 & 1.43 & 36.97 & 2.43 \\
\hline \multirow[t]{3}{*}{ ES } & & & 8.5 & 20.04 & 8.49 & 21.22 & 1.19 & 0.35 & 8.49 & 5.95 & 1.49 & 31.47 & 2.09 \\
\hline & \multicolumn{9}{|c|}{ Eq. (5) } & & & & \\
\hline & $\rho_{1}$ & $\rho_{2}$ & $\alpha_{\mathrm{i}}$ & $\beta_{1}$ & $\gamma_{1}$ & $\beta_{2}$ & $\beta_{2}$ & $\beta_{3}$ & $\gamma_{3}$ & & & & \\
\hline ES & 0.15 & 0.18 & 8.5 & 2.76 & 1.77 & 7.82 & 3.34 & 31.04 & 2.05 & & & & \\
\hline
\end{tabular}


same as the mean DBH and its standard deviation for each group and entire stand respectively. There was a difference among the scales and shape parameters in eqs. (2)-(5), when the values of different locations were used. All scales and shape parameters in
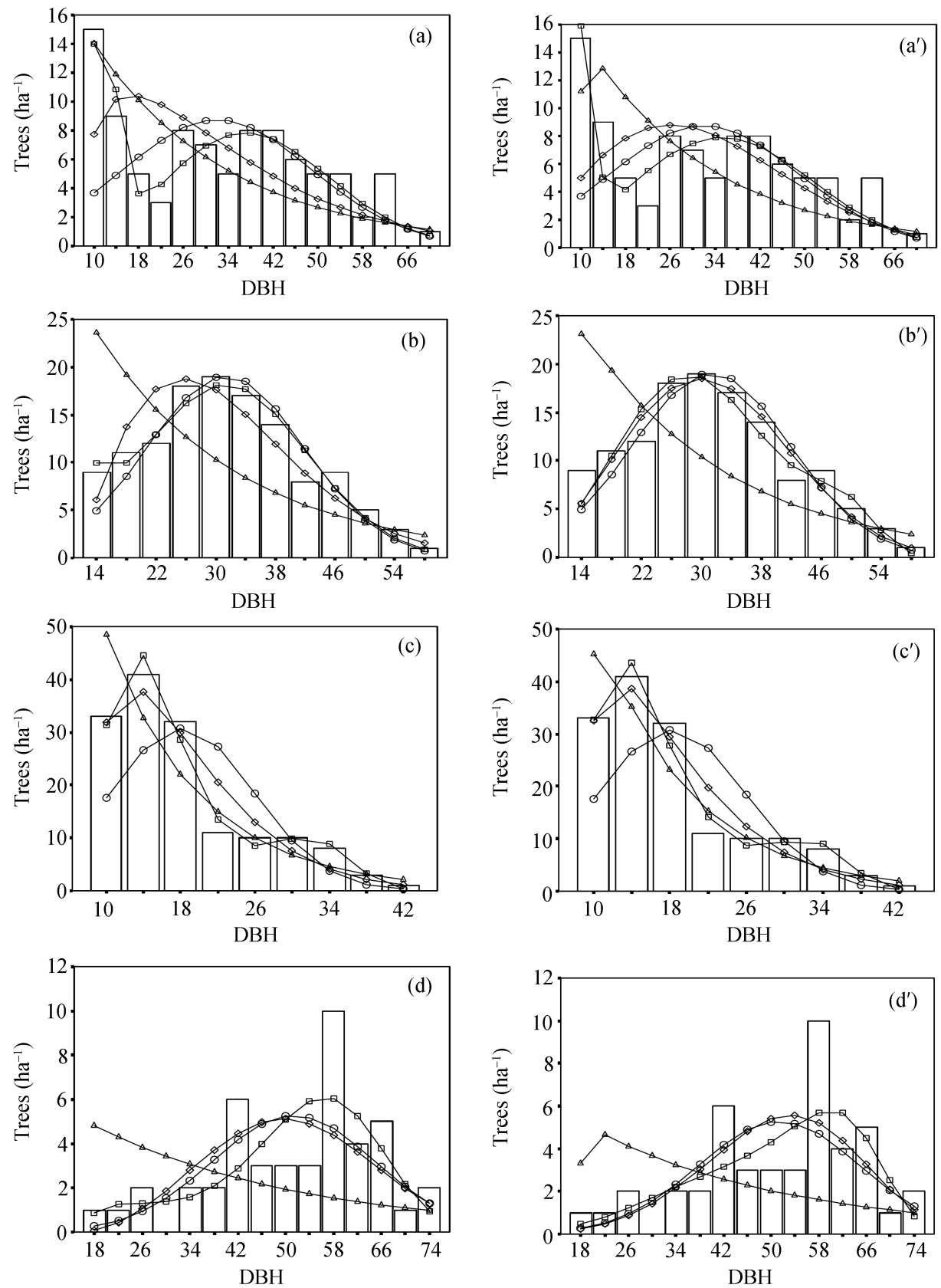

Fig. 1. Model comparison for S1, S2, S3 and S4. The histogram represents the observed diameter distribution and the lines show the estimated distributions with model $1(\bigcirc)$, model $2(\triangle)$, model $3(\diamond)$, model $4(\square)$, model $5(\nabla)$ for (a) S1, (b) S2, (c) S3, (d) S4 when parameters in Table 2(a) were used and for (a') S1, (b') S2, (c') S3, (d') S4 when parameters in Table 2(b) were used. 
eqs. (2) - (5) were bigger than that in eq. (1) except the scale parameter of S2 in eq. (4) with a low value location parameter in the smallest diameter class. The scale parameters increased gradually in eqs. (4) and (5).

\subsection{Model comparison}

The observed and estimated frequency distribution of each group and entire stand are shown in Figs. 1-3 and Table 2. The histograms showed the frequencies by $4 \mathrm{~cm}$ diameter classes of each group and entire stand except S6. The frequency at S6 was shown by $1 \mathrm{~cm}$ diameter class due to narrow diameter class range. The curves represent the estimated distributions. It revealed that eq. (1) was suitable for S2, S4, S6, S7 and CTS, eq. (2) was the only one suitable for S6, eq. (3) was fitted for S2, S3, S6, CTS, eq. (4) met
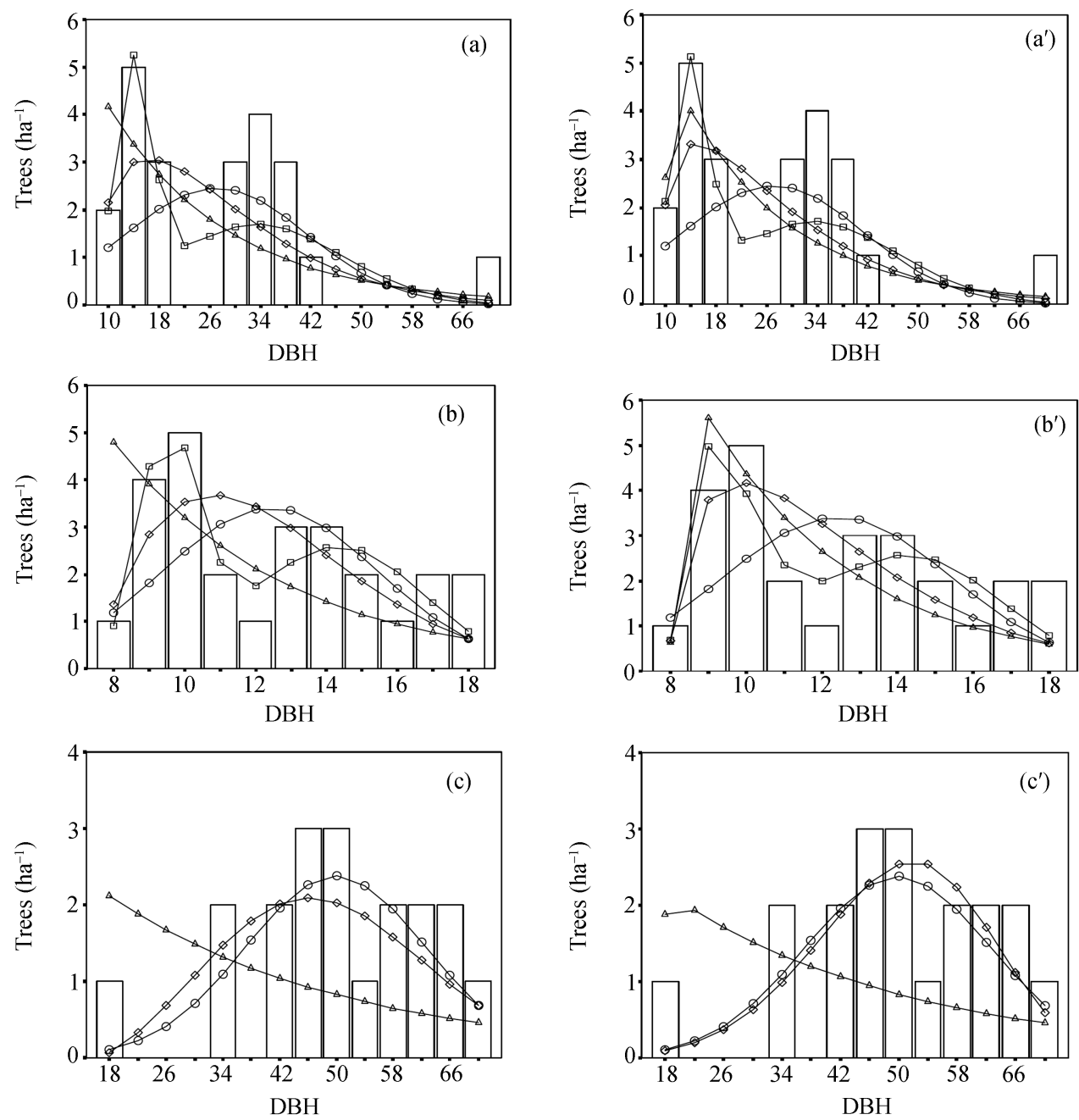

Fig. 2. Model comparison for S5, S6 and S7. The histogram represents the observed diameter distribution and the lines show the estimated distributions with model $1(\bigcirc)$, model $2(\triangle)$, model $3(\diamond)$, model $4(\square)$, model $5(\nabla)$ for (a) S5, (b) S6, (c) S7 when parameters in Table 2(a) were used and for (a') S5, (b') S6, (c') S7 when parameters in Table 2(b) were used. 

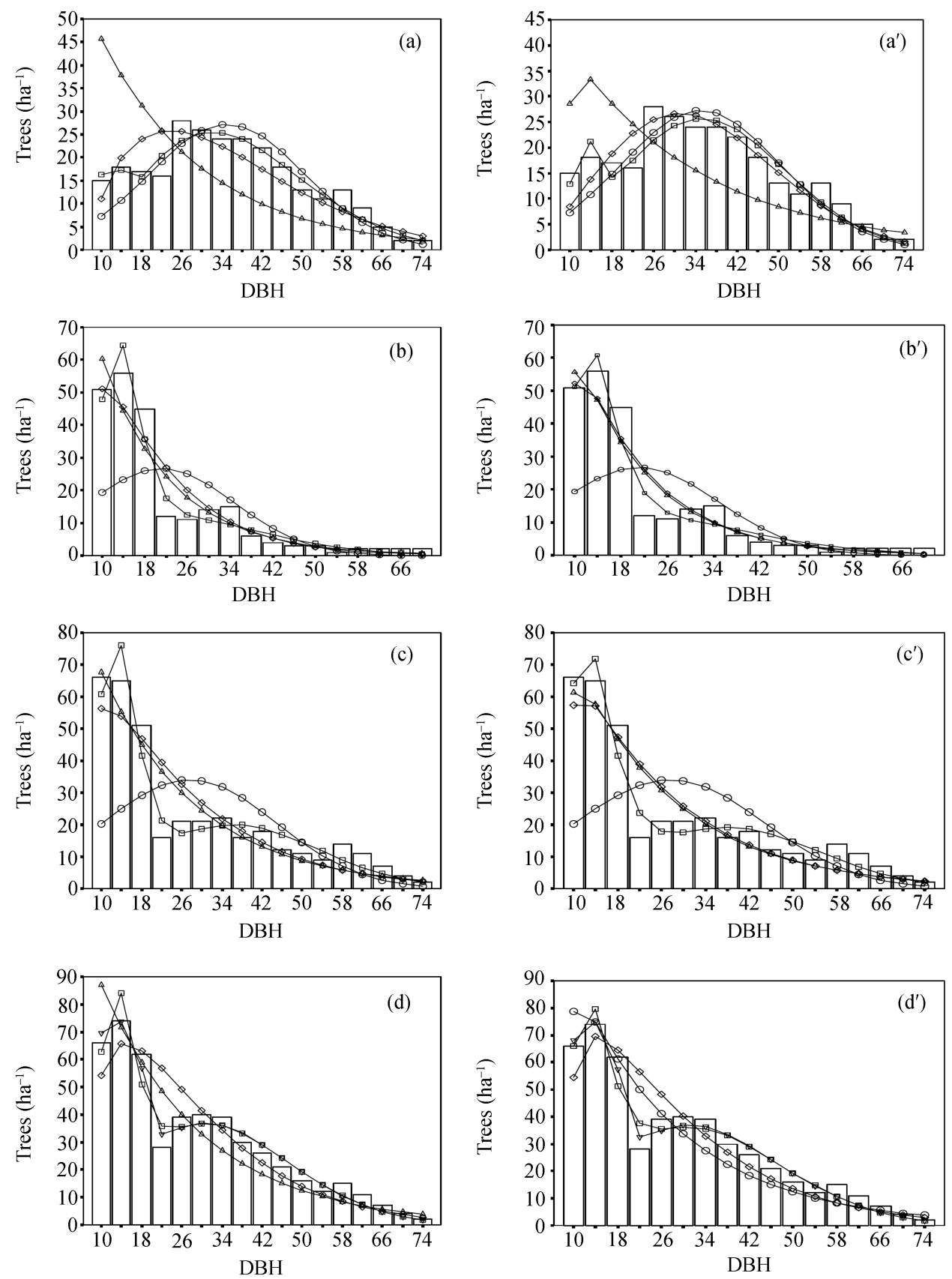

Fig. 3. Model comparison for CTS, SCTS, BTS and ES. The histogram represents the observed diameter distribution and the lines show the estimated distributions with model $1(\bigcirc)$, model $2(\triangle)$, model $3(\diamond)$, model $4(\square)$, model $5(\nabla)$ for (a) TS, (b) SCTS, (c) BTS, (d) ES when parameters in Table 2(a) were used and for (a') CTS, (b') SCTS, (c') BTS, (d') ES when parameters in Table 2(b) were used. 
S1, S2, S3, S4, S6, CTS, BTS and ES, and eq. (5) was fitted for ES. In addition, eq. (3) was also fitted for S4 and S7, when the parameters in Table 2(b) were used, based on the $p$. By comparing the results with different parameters, there was a difference based on the RMSE and Bias.

Table 2 The root mean square error (RMSE), bias (Bias) and $x^{2}$ test of the fitting methods for each group and entire stand with (a) the parameters in Table 2(a) used and (b) the parameters in Table 2(b) used

(a)

\begin{tabular}{|c|c|c|c|c|c|c|c|c|c|c|c|c|}
\hline & \multicolumn{3}{|c|}{ Eq. (1) Eq. (5) } & \multicolumn{3}{|c|}{ Eq. (2) } & \multicolumn{3}{|c|}{ Eq. (3) } & \multicolumn{3}{|c|}{ Eq. (4) } \\
\hline & RMSE & Bias & $x^{2}$ & RMSE & Bias & $x^{2}$ & RMSE & Bias & $x^{2}$ & RMSE & Bias & $x^{2}$ \\
\hline $\mathrm{S} 1$ & 3.49 & 0.48 & $50.59 *$ & 2.88 & 0.40 & $31.27^{*}$ & 3.29 & 0.22 & $29.61 *$ & 1.44 & 0.04 & 9.66 \\
\hline S2 & 1.97 & 0.37 & 6.93 & 6.79 & 0.87 & $46.40^{*}$ & 2.39 & 0.15 & 6.31 & 1.40 & 0.04 & 2.53 \\
\hline S3 & 9.44 & 1.52 & $44.86^{*}$ & 7.03 & 0.48 & $17.18^{*}$ & 3.93 & 0.11 & 10.32 & 2.03 & 0.00 & 3.01 \\
\hline S4 & 1.95 & 0.12 & 17.65 & 3.18 & 0.55 & $80.77^{*}$ & 2.03 & 0.11 & $29.76^{*}$ & 1.78 & 0.03 & 12.84 \\
\hline S5 & 1.42 & 0.12 & $53.84 *$ & 1.41 & 0.05 & $24.84 *$ & 1.35 & 0.01 & $23.11 *$ & 1.01 & 0.00 & $30.20 *$ \\
\hline S6 & 1.39 & 0.18 & 11.34 & 1.58 & 0.25 & 13.13 & 1.20 & 0.09 & 8.06 & 0.66 & 0.05 & 3.46 \\
\hline S7 & 0.77 & 0.86 & 13.49 & 1.41 & 3.64 & $29.80 *$ & 0.87 & 1.08 & $19.72 *$ & & & \\
\hline CTS & 3.71 & 0.67 & 22.30 & 11.85 & 0.60 & $126.00^{*}$ & 3.88 & 0.49 & 15.63 & 2.07 & 0.17 & 5.58 \\
\hline SCTS & 13.66 & 2.42 & $263.05^{*}$ & 6.14 & 0.11 & $27.56^{*}$ & 5.76 & 0.05 & $32.05^{*}$ & 4.05 & 0.05 & $19.33^{*}$ \\
\hline BTS & 17.56 & 2.77 & $244.20 *$ & 6.89 & 0.67 & $43.14 *$ & 8.01 & 0.49 & $48.77 *$ & 4.85 & 0.16 & 17.43 \\
\hline ES & (3.25) & $(0.14)$ & $(9.31)$ & 8.78 & 1.06 & $39.29 *$ & 8.64 & 0.47 & $32.11 *$ & 4.95 & 0.14 & 13.41 \\
\hline
\end{tabular}

\begin{tabular}{|c|c|c|c|c|c|c|c|c|c|c|c|c|}
\hline & \multicolumn{3}{|c|}{ Eq. (1) \& Eq. (5) } & \multicolumn{3}{|c|}{ Eq. (2) } & \multicolumn{3}{|c|}{ Eq. (3) } & \multicolumn{3}{|c|}{ Eq. (4) } \\
\hline & RMSE & Bias & $x^{2}$ & RMSE & Bias & $x^{2}$ & RMSE & Bias & $x^{2}$ & RMSE & Bias & $x^{2}$ \\
\hline S1 & 3.49 & 0.48 & $50.59 *$ & 3.20 & 0.38 & $33.09 *$ & 3.32 & 0.35 & $35.70^{*}$ & 1.71 & 0.12 & 12.39 \\
\hline S2 & 1.97 & 0.37 & 6.93 & 6.71 & 0.87 & $45.69^{*}$ & 1.63 & 0.22 & 4.29 & 1.62 & 0.15 & 4.55 \\
\hline S3 & 9.44 & 1.52 & $44.86^{*}$ & 5.81 & 0.42 & $13.74 *$ & 3.62 & 0.13 & 9.56 & 2.06 & 0.00 & 3.70 \\
\hline S4 & 1.95 & 0.12 & 17.65 & 3.12 & 0.53 & $76.66^{*}$ & 1.88 & 0.06 & 16.72 & 1.71 & 0.03 & 13.27 \\
\hline S5 & 1.42 & 0.12 & $53.84 *$ & 5.20 & 0.04 & $23.03 *$ & 5.34 & 0.02 & $22.40^{*}$ & 4.06 & 0.00 & $30.13^{*}$ \\
\hline S6 & 1.39 & 0.18 & 11.34 & 1.15 & 0.19 & 9.79 & 1.12 & 0.12 & 8.03 & 0.80 & 0.05 & 4.21 \\
\hline S7 & 0.77 & 0.86 & 13.49 & 1.41 & 0.26 & $29.19^{*}$ & 0.78 & 0.03 & 15.1 & & & \\
\hline CTS & 3.71 & 0.67 & 22.30 & 8.44 & 1.18 & $73.51^{*}$ & 3.04 & 0.33 & 13.48 & 2.69 & 0.20 & 8.61 \\
\hline SCTS & 13.66 & 2.42 & $263.05^{*}$ & 5.42 & 0.09 & $25.95^{*}$ & 5.36 & 0.07 & $27.77 *$ & 3.92 & 0.05 & $20.08 *$ \\
\hline BTS & 17.56 & 2.77 & $244.20^{*}$ & 7.03 & 0.63 & $44.75^{*}$ & 7.53 & 0.56 & $47.14 *$ & 4.43 & 0.14 & 16.91 \\
\hline ES & (3.18) & $(0.14)$ & $(9.28)$ & 7.90 & 1.00 & $36.27 *$ & 8.46 & 0.54 & $32.23 *$ & 4.66 & 0.14 & 13.22 \\
\hline
\end{tabular}

The number in the parentheses shows the result for eq. (5), ${ }^{*} p<0.05$.

\section{Discussions}

In the broadleaved-Korean pine mixed forest on the Changbai Mountains of China, the diameter distributions can be grouped into two categories: one includes S2, S4, S7 and CTS which belongs to normal distributions; the other can be merged into "S" curve. For species, the different diameter distributions reveal the regeneration patterns, continuous and discontinuous, as indicated by Saxena ${ }^{[16]}$. S1, S3 and so on belong to the former category, the normal distribution including S2, S4 and S7 comports with the latter.

The relations between estimated parameters and stand characteristics such as stand age, mean DBH, etc, have been discussed. In Larix plantation stand in Liaoning Province of China, the parameters in the Weibull presented positive correlations with height and mean DBH, and negative correlation with stand density ${ }^{[17]}$. Nanang ${ }^{[18]}$ thought that the 
parameters in the Weibull can be estimated by stand age, mean DBH and height in plantation stand in Northern Ghana. In this paper, the parameters in eq. (1) were respectively equal to the mean DBH and standard deviation. These results from the characteristics of maximum likelihood estimation of the normal equation, i.e. $\mu$ and $\sigma$ in normal distribution were the mean and standard deviation in the maximum likelihood equation, respectively. The location parameters of all equations in Table 1(a) reached a significant positive relation with min DBH $(R=0.989, p=0.00, n=11)$ and mean DBH $(R=0.838$, $p=0.01, n=11$ ), because the location parameter was subjectively considered as a low value in the smallest diameter class. The location parameter of eq. (2) in Table 1(b) also reached a significant positive relation with $\min \mathrm{DBH}(R=1, p=0.00, n=11)$ and mean $\mathrm{DBH}(R=0.856, p=0.01, i=11)$, which was correlated with the soft package. The location parameters of eqs. (3) $-(5)$ in Table $1(b)$ reached significant negative relations with min DBH $(R=-0.675, p=0.023, n=11)$ and mean DBH $(R=-0.829, p=0.002, n=11)$. The positive relation between the location parameter and min DBH and mean DBH could relate with the arbitrary choice based on the math ${ }^{[17,18]}$. The scale parameters in eq. (2) positively related with mean DBH. Their correlations reached respectively $0.980(p=0.000$, $n=11)$ and $0.981(p=0.000, n=11)$ with the scale parameters in Table 1(a) and (b). The scale parameters in eq. (3) positively related with mean $\mathrm{DBH}$, and their correlations reached respectively $0.981(p=0.000, n=11)$ and $0.983(p=0.000, n=11)$ with the scale parameters in Table 1(a) and (b). The first scale parameter in eq. (4) positively related with mean DBH, and their correlations reached respectively $0.723(p=0.018, n=10)$ and $0.844(p=0.002, n=10)$ with the scale parameters in Table 1(a) and (b). The second scale parameters in eq. (4) positively related with mean $\mathrm{DBH}$, and their correlations reached respectively $0.828(p=0.003, n=10)$ and $0.960(p=0.000, n=10)$ with the scale parameters in Table 1(a) and (b). The shape parameters in eq. (3) reached significant positive correlations with mean DBH, and their correlations were respectively $0.763(p=0.006, n=11)$ and $0.900(p=0.000, n=11)$ with the scale parameters in Table 1(a) and (b). The first shape parameters in eq. (4) had no correlation with mean $\mathrm{DBH}$, and their correlations reached only respectively $-0.491(p=0.15, n=10)$ and $0.372(p=0.289, n=10)$ with the scale parameters in Table 1(a) and (b). The second scale parameters in eq. (4) had no correlation with mean $\mathrm{DBH}$, and their correlations were respectively $0.033(p=0.929$, $n=10)$ and $0.421(p=0.226, n=10)$ with the scale parameters in Table 1(a) and (b). The correlations between the parameters including location parameters, scale parameters and shape parameters and mean DBH were greater when the location parameter was estimated by the soft package compared with that by man. This results from the fact that the parameters were directly correlated with observed data in maximum likelihood equations of these equations. The high correlations between the parameter and mean DBH made the parameter estimated by the mean DBH possible, but the shape parameters in the mixture distribution were too complex to be characterized by the mean DBH.

The flexibilities of two-parameter negative exponential equation and three-parameter Weibull function were embodied. There were 8 RMSEs with two-parameter negative exponential equation smaller than one-parameter. There were 9 RMSEs with three-pa- 
rameter Weibull function smaller than two-parameter. The results were the same as negative exponential, and different from Weibull distribution, based on the Bias. There was no obvious difference, when the location parameters in the mixture distribution were estimated by different methods. The result may come from both location parameters in the mixture distribution not estimated by its maximum likelihood equation.

The Weibull distribution with three-parameter was so flexible, which could be characterized by the normal distribution, based on the $\chi^{2}$, and was better than the normal distribution in accuracy based on the RMSE and Bias (Table 2(b)). The shape and accuracy in mixture distribution of two Weibull functions was much more flexible than that in a Weibull function. The Weibull function only was able to describe the unimodal curve, however the mixture distribution was able to characterize the unimodal and multimodal curve (Table 2). In theory, the mixture distribution of two Weibull functions can characterize the three-modal curve, but the mixture distribution only can describe two-modal curve when there are three modals in the observed frequency such as BTS, ES. Some distributions such as the S5 were not characterized by the mixture distribution of two Weibull functions.

The component in the mixture distribution was discussed in the plantation and two-species mixed forest stands ${ }^{[14,15]}$. The first component in the mixture of two Weibull distributions describing ES was fitted for BTS and BCTS in shape but the accuracy was worse than the single Weibull based on the RMSE and Bias. The second component in the mixture of two Weibull distributions describing the ES was fitted for S2 and CTS in shape but the accuracy was worse than the single Weibull based on the RMSE and Bias. This may relate with the used social class, or this may result from the characteristics of the mixture function. The estimated frequency was only obtained from math not biology. The estimated frequencies of the ES can be obtained by characterizing the ES or summing up the frequencies of species group. By comparing two methods, the estimated fre-

Table 3 The RMSE and Bias of the observed and estimated frequency with the different equation combinations in the ES by $4 \mathrm{~cm}$ diameter class for S2 vs. BTS (a) and CTS and SCTS (b) using the parameters in Table 2(a)

(a)

\begin{tabular}{|c|c|c|c|c|c|c|c|c|}
\hline \multirow{3}{*}{ BTS } & \multicolumn{8}{|c|}{$\mathrm{S} 2$} \\
\hline & \multicolumn{2}{|c|}{ Eq. (1) } & \multicolumn{2}{|c|}{ Eq. (2) } & \multicolumn{2}{|c|}{ Eq. (3) } & \multicolumn{2}{|c|}{ Eq. (4) } \\
\hline & RMSE & Bias & RMSE & Bias & RMSE & Bias & RMSE & Bias \\
\hline Eq. (1) & 18.4621 & 3.0361 & 14.4685 & 3.3897 & 17.9314 & 2.8811 & 17.5602 & 2.8009 \\
\hline Eq. (2) & 7.5055 & 0.9298 & 7.7521 & 1.2835 & 8.1968 & 0.7748 & 6.8683 & 0.6946 \\
\hline Eq. (3) & 8.5936 & 0.7520 & 8.3070 & 1.1056 & 9.2453 & 0.5970 & 7.9698 & 0.5168 \\
\hline Eq. (4) & 4.8693 & 0.4184 & 8.2387 & 0.7720 & 4.6540 & 0.2633 & 5.2924 & 0.1832 \\
\hline \multicolumn{9}{|l|}{ (b) } \\
\hline \multirow{3}{*}{ SCTS } & \multicolumn{8}{|c|}{ CTS } \\
\hline & \multicolumn{2}{|c|}{ Eq. (1) } & \multicolumn{2}{|c|}{ Eq. (2) } & \multicolumn{2}{|c|}{ Eq. (3) } & \multicolumn{2}{|c|}{ Eq. (4) } \\
\hline & RMSE & Bias & RMSE & Bias & RMSE & Bias & RMSE & Bias \\
\hline Eq. (1) & 15.9423 & 2.9406 & 8.6350 & 2.8776 & 13.7156 & 2.7650 & 13.4874 & 2.4471 \\
\hline Eq. (2) & 7.3430 & 0.7675 & 13.1983 & 0.7044 & 6.7059 & 0.5919 & 6.8106 & 0.2740 \\
\hline Eq. (3) & 7.5180 & 0.7161 & 12.0125 & 0.6530 & 7.0815 & 0.5405 & 6.3874 & 0.2226 \\
\hline Eq. (4) & 5.4193 & 0.7139 & 12.4547 & 0.6508 & 5.5290 & 0.5383 & 4.6816 & 0.2204 \\
\hline
\end{tabular}


quencies of S2 vs. BTS and CTS vs. SCTS were summed up and the RMSE and Bias were computed (Table 3). There were only three RMSEs smaller than 4.9484 in 32 combinations.

The location parameter had an effect on the estimated frequency in the first diameter class when the estimated location parameter was greater than the lower limit of the first diameter class. For example, the cumulative equation of the negative exponential distribution was: $F(x)=\mathrm{e}^{-\frac{x-\alpha}{\beta}}$, where $\alpha$ and $\beta$ are the location and scale. That $F\left(x_{1}\right)$ where $x_{1}$ was the lower limit of the first diamecer class was $>1$ when the estimated location parameter was greater than $x_{1}$ conflicted with the math property, so $F\left(x_{1}\right)$ was set as 1 . Then the probability decreased in the first diameter class, so the frequency in the first diameter class was underestimated. The distortion appeared such as the negative exponential distribution in Figs. 1-3 when the parameters in Table 2(b) were used. The distortion also produced the error in the negative exponential distribution for S6.

\section{Conclusions}

The diameter classes of species and entire stand showed not negative exponential but normal and "S" distributions. The parameters in all equations reached very high correlations with mean DBH, except the shape parameters in the mixture distribution. The mixture function was so flexible that it characterized various diameter class distributions, and it was better than normal and Weibull in shape and accuracy. However, the mixture function of two components only described two-modal curve, which cannot characterize especial "S" curve such as S5. Whether the location parameter was subjectively chosen based on the math or not would influence the flexibility of the negative exponential and Weibull distribution, the location parameter had an effect on the estimated frequency in the first diameter class, when the estimated location parameter was bigger than the lower limit of the first diameter class.

Acknowledgements This work was jointly supported by the National Natural Science Foundation of China (Grant Nos. 70373044 and 30470302), China's Ministry of Science and Technology (04EFN216600328), and the Northeast Rejuvenation Program of the Chinese Academy of Sciences. We thank the staff at the Changbai Research Station who helped us obtain the observed data and Prof. Guofan Shao of Purdue University for his valuable advice on this research.

\section{References}

1 Bailey R L, Dell T R. Quantifying diameter distributions with the Weibull function. For Sci, 1973, 19: 97104

2 Pierrat J C, Herve J C, Peyron J L. Modelling of the structure of a stand and simulation of the local and selective thinning strategies. Can J For Res, 1997, 27(6): 840-848

3 Guan D X, Wu J B, Yu G R, et al. Meteorological control on $\mathrm{CO}^{2}$ flux above broad-leaved Korean pine mixed forest in Changbai Mountains. Sci China Ser D-Earth Sci, 2005, 48 (supp. I): 116-122

4 Wu J B, Guan D X, Sun X M, et al. Eddy flux corrections for $\mathrm{CO}^{2}$ exchange in broad-leaved Korean pine mixed forest of Changbai Mountains. Sci China Ser D-Earth Sci, 2005, 48 (supp. I): 106-115 
5 Gu H Y, Dai L M, Wu G, et al. Estimation of forest volumes by integrating Landsat TM imagery and forest inventory data. Sci China Ser E, 2006, 49 (supp. I): 54-62

6 Dai L M, Liu Q J, Zhang Y P, et al. Structure and changes of broad-leaved-conifer-Korean pine mixed forest in Northeast China. J Forest Res, 1998, 9(3): 141-146

7 Meyer H A, Stevenson D D. The structure and growth of virgin beech-birch-maple-hemlock forests in northern Pennsylvania. J Agric Res, 1943, 67: 465-484

8 Meyer H A. Structure, growth, and drain in balanced uneven-aged forests. J For, 1952, 52: 85-92

9 Schmelz D V, Lindsey A A. Size-class structure of old-growth forests in Indian. For Sci, 1965, 11: 258-264

10 Leak W B. The shaped probability distribution. For Sci, 1965, 11: 405-409

11 Weibull W. A statistical distribution function of wide applicability. J Appl Mech, 1951, 18: 293 - 297

12 Gogg F G, West D. Canopy-understory interaction effects on forest population structure. For Sci, 1975, 21: 98 $-108$

13 Zhang L J, Gove J H, Liu C M, et al. A finite mixture of two Weibull distributions for modeling the diameter distributions of rotated-sigmoid, uneven-aged stands. Can J For Res, 2001, 31: 1654-1659

14 Liu C M, Zhang L J, Davig C J, et al. A finite mixture model for characterizing the diameter distributions of mixed-species forest stands. For Sci, 2002, 48(4): 653-661

15 Zasada M, Cieszewski C J. A finite mixture distribution approach for characterizing tree diameter distributions by natural social class in pure even-aged Scots pine stands in Poland. For Ecol Manage, 2005, 204: 145-158

16 Saxena A K, Singh J S. Tree population structure of certain Himalayan forest associations and implications concerning their future composition. Plant Ecol, 1984, 58: 61-69

17 Liu J R, Zhao D F. Weibull distribution parameters and stand factor model of Larix plantation. Sci Silvae Sin (in Chinese), 1997, 33(5): 412-417

18 Nanang D M. Suitability of the normal, log-normal and Weibull distributions for fitting diameter distributions of neem plantations in Northern Ghana. For Ecol Manage, 1998, 103: 1-7 